\title{
RPC system in the CMS Level-1 Muon Trigger
}

To cite this article: A. Samalan et al 2020 JINST 15 C10007

View the article online for updates and enhancements.

\section{IOP ebooks}

Bringing together innovative digital publishing with leading authors from the global scientific community. Start exploring the collection-download the first chapter of every title for free. 


\section{RPC system in the CMS Level-1 Muon Trigger}

A. Samalan ${ }^{a}$ M. Tytgat, ${ }^{a}$ N. Zaganidis, ${ }^{a}$ G.A. Alves, ${ }^{b}$ F. Marujo, ${ }^{b}$

F. Torres Da Silva De Araujo, ${ }^{c}$ E.M. Da Costa, ${ }^{c}$ D. De Jesus Damiao, ${ }^{c}$ H. Nogima, ${ }^{c}$

A. Santoro, ${ }^{c}$ S. Fonseca De Souza, ${ }^{c}$ A. Aleksandrov,${ }^{d}$ R. Hadjiiska, ${ }^{d}$ P. laydjiev, ${ }^{d}$

M. Rodozov, ${ }^{d}$ M. Shopova, ${ }^{d}$ G. Sultanov, ${ }^{d}$ M. Bonchev, ${ }^{e}$ A. Dimitrov, ${ }^{e}$ L. Litov, ${ }^{e}$ B. Pavlov, ${ }^{e}$

P. Petkov, ${ }^{e}$ A. Petrov, ${ }^{e}$ S.J. Qian, ${ }^{f}$ P. Cao, ${ }^{b b}$ H. Kou, ${ }^{b b}$ Z. Liu, ${ }^{b b}$ J. Song, ${ }^{b b}$ J. Zhao, ${ }^{b b}$

C. Bernal, ${ }^{g}$ A. Cabrera,${ }^{g}$ J. Fraga, ${ }^{g}$ A. Sarkar,${ }^{g}$ S. Elsayed,${ }^{h}$ Y. Assran, ${ }^{c c, d d}$ M. El Sawy, ${ }^{c c, e e}$ M.A. Mahmoud, ${ }^{i}$ Y. Mohammed, ${ }^{i}$ C. Combaret,${ }^{j}$ M. Gouzevitch,${ }^{j}$ G. Grenier,${ }^{j}$ I. Laktineh,${ }^{j}$ L. Mirabito, ${ }^{j}$ K. Shchablo, ${ }^{j}$ I. Bagaturia, ${ }^{k}$ D. Lomidze,${ }^{k}$ I. Lomidze,${ }^{k}$ V. Bhatnagar, ${ }^{l}$ R. Gupta, ${ }^{l}$ P. Kumari, ${ }^{l}$ J. Singh, ${ }^{l}$ V. Amoozegar, ${ }^{m}$ B. Boghrati, ${ }^{m}$ M. Ebraimi, ${ }^{m}$ R. Ghasemi, ${ }^{m}$ M. Mohammadi Najafabadi, ${ }^{m}$ E. Zareian, ${ }^{m}$ M. Abbrescia, ${ }^{n}$ R. Aly, ${ }^{n}$ W. Elmetenawee, ${ }^{n}$ N. De Filippis, ${ }^{n}$ A. Gelmi, ${ }^{n}$ G. laselli, ${ }^{n}$ S. Leszki, ${ }^{n}$ F. Loddo, ${ }^{n}$ I. Margjeka, ${ }^{n}$ G. Pugliese,${ }^{n}$ D. Ramos, ${ }^{n}$ L. Benussi, ${ }^{o}$ S. Bianco, ${ }^{o}$ D. Piccolo, ${ }^{o}$ S. Buontempo, ${ }^{p}$ A. Di Crescenzo, ${ }^{p}$ F. Fienga, ${ }^{p}$ G. De Lellis,${ }^{p}$ L. Lista, ${ }^{p}$ S. Meola, ${ }^{p}$ P. Paolucci, ${ }^{p}$ A. Braghieri, ${ }^{q}$ P. Salvini, ${ }^{q}$ P. Montagna, ${ }^{f f}$ C. Riccardi, ${ }^{f f}$ P. Vitulo, ${ }^{f f}$ J.E. Choi, ${ }^{r}$ B. Francois, ${ }^{r}, 1$ T.J. Kim, ${ }^{r}$ J. Park, ${ }^{r}$ S.Y. Choi, ${ }^{s}$ B. Hong, ${ }^{s}$ K.S. Lee, ${ }^{s}$ J. Goh, ${ }^{t}$ H. Lee, ${ }^{u}$ J. Eysermans, ${ }^{v}$ C. Uribe Estrada, ${ }^{v}$ I. Pedraza, ${ }^{v}$ H. Castilla-Valdez, ${ }^{w}$ A. Sanchez-Hernandez, ${ }^{w}$ C.A. Mondragon Herrera, ${ }^{w}$ D.A. Perez Navarro, ${ }^{w}$ G.A. Ayala Sanchez, ${ }^{w}$ S. Carrillo, ${ }^{x}$ E. Vazquez, ${ }^{x}$ A. Radi, ${ }^{y}$ A. Ahmad, ${ }^{z}$ I. Asghar, ${ }^{z}$ H. Hoorani, ${ }^{z}$ S. Muhammad, ${ }^{z}$ M.A. Shah ${ }^{z}$ and I. Crotty ${ }^{a a}$ on behalf of the CMS Collaboration

${ }^{a}$ Ghent University, Department of Physics and Astronomy, Proeftuinstraat 86, B-9000 Ghent, Belgium

${ }^{b}$ Centro Brasileiro Pesquisas Fisicas,

R. Dr. Xavier Sigaud, 150 - Urca, Rio de Janeiro - RJ, 22290-180, Brazil

${ }^{c}$ Departamento de Fisica Nuclear e Altas Energias, Instituto de Fisica,

Universidade do Estado do Rio de Janeiro,

Rua Sao Francisco Xavier, 524, BR - Rio de Janeiro 20559-900, RJ, Brazil

${ }^{d}$ Bulgarian Academy of Sciences, Institutes for Nuclear Research and Nuclear Energy,

Tzarigradsko shaussee Boulevard 72, BG-1784 Sofia, Bulgaria

${ }^{e}$ Faculty of Physics, University of Sofia, 5 James Bourchier Boulevard, BG-1164 Sofia, Bulgaria

${ }^{f}$ School of Physics, Peking University, Beijing 100871, China

${ }^{1}$ Corresponding author. 
${ }^{g}$ Universidad de Los Andes, Apartado Aereo 4976, Carrera 1E, no. 18A 10, CO-Bogota, Colombia

${ }^{h}$ Egyptian Network for High Energy Physics, Academy of Scientific Research and Technology,

101 Kasr El-Einy St. Cairo Egypt

${ }^{i}$ Center for High Energy Physics, Faculty of Science, Fayoum University, 63514 El-Fayoum, Egypt

${ }^{j}$ Université Lyon, Université Claude Bernard Lyon 1, CNRS/IN2P3, IP2I Lyon, UMR 5822,

F-69622, Villeurbanne, France

${ }^{k}$ Georgian Technical University, 77 Kostava Str., Tbilisi 0175, Georgia

${ }^{l}$ Department of Physics, Panjab University, Chandigarh 160 014, India

${ }^{m}$ School of Particles and Accelerators, Institute for Research in Fundamental Sciences (IPM),

P.O. Box 19395-5531, Tehran, Iran

${ }^{n}$ INFN, Sezione di Bari, Via Orabona 4, IT-70126 Bari, Italy

${ }^{\circ}$ INFN, Laboratori Nazionali di Frascati (LNF), Via Enrico Fermi 40, IT-00044 Frascati, Italy

p INFN, Sezione di Napoli, Complesso Universitario Monte S. Angelo, Via Cintia, IT-80126 Napoli, Italy

q INFN, Sezione di Pavia, Via Bassi 6, IT-Pavia, Italy

${ }^{r}$ Hanyang University, 222 Wangsimni-ro, Sageun-dong, Seongdong-gu, Seoul, Republic of Korea

${ }^{s}$ Korea University, Department of Physics, 145 Anam-ro, Seongbuk-gu, Seoul 02841, Republic of Korea

${ }^{t}$ Kyung Hee University, 26 Kyungheedae-ro, Hoegi-dong, Dongdaemun-gu, Seoul, Republic of Korea

"Sungkyunkwan University,

2066 Seobu-ro, Jangan-gu, Suwon, Gyeonggi-do 16419, Seoul, Republic of Korea

${ }^{v}$ Benemerita Universidad Autonoma de Puebla, Puebla, Mexico

${ }^{w}$ Cinvestav,

Av. Instituto Politécnico Nacional No. 2508, Colonia San Pedro Zacatenco, CP 07360 ,

Ciudad de Mexico D.F., Mexico

${ }^{x}$ Universidad Iberoamericana, Mexico City, Mexico

${ }^{y}$ Sultan Qaboos University, Al Khoudh,Muscat 123, Oman

${ }^{z}$ National Centre for Physics, Quaid-i-Azam University, Islamabad, Pakistan

${ }^{a}$ Department of Physics, Wisconsin University, Madison, WI 53706, United States

${ }^{b b}$ Institute of High Energy Physics, UCAS/CAS, Beijing, China

${ }^{c c}$ The British University in Egypt (BUE),

Elsherouk City, Suez Desert Road, Cairo 11837 - P.O. Box 43, Egypt

${ }^{d d}$ Suez University, Elsalam City, Suez - Cairo Road, Suez 43522, Egypt

ee Department of Physics, Faculty of Science, Beni-Suef University, Beni-Suef, Egypt

ff INFN, Sezione di Pavia and University of Pavia, Via Bassi 6, IT-Pavia, Italy

E-mail: brieuc.francois@cern.ch

Abstract: The CMS experiment implements a two-level triggering system composed of Level-1, instrumented by custom-design hardware boards, and a software High Level Trigger. To cope with the more challenging luminosity conditions, a new Level-1 architecture has been deployed during run II. This new architecture exploits in a better way the redundancy and complementarity of the three muon subsystems: Cathode Strip Chambers (CSC), Drift Tubes (DT) and Resistive Plate Chambers (RPC). The role of each subsystem in the Level-1 Muon Trigger is described here, highlighting the contribution from the RPC system. Challenges brought by the HL-LHC environment and new possibilities coming from detector and trigger upgrades are also discussed.

Keywords: Resistive-plate chambers; Trigger concepts and systems (hardware and software); Trigger detectors 


\section{Contents}

1 Introduction 1

2 Muon system and legacy Level-1 Muon Trigger 1

$3 \quad$ RPC in Run 2 Level-1 Muon Trigger 3

4 RPC in Phase 2 Level-1 Muon Trigger 4

5 Conclusion 5

\section{Introduction}

One of the big challenges we face in modern high energy physics is to be able to study rare processes. These rare processes occur at rates which are orders of magnitude smaller than the inclusive rate of proton-proton interactions (e.g. ten orders of magnitude smaller for the production of the Higgs boson). To ensure having enough data to study such processes, the Large Hadron Collider (LHC) operates with high intensity beams and a proton-proton collision rate of $40 \mathrm{MHz}$. Bandwidth, storage and computing power are currently not sufficient to record all these collisions, forcing us to apply a selection. The Compact Muon Solenoid (CMS) [1] selects potentially interesting events through a two-level triggering system. The Level-1 Trigger (L1T) processes collisions at the full LHC collision rate $(40 \mathrm{MHz})$, using coarse resolution information from calorimeters and muon detectors. The design of the system is based on custom hardware, selecting one event out of four hundred and takes the decision in $3.6 \mu \mathrm{s}$. The selected events are then passed to the High Level Trigger (HLT) which further applies a rate reduction of a factor one hundred based on the full detector information and using commercial server farms. Muons play a significant role in the selection of potentially interesting events as they are involved in many extensions of the Standard Model (SM) as well as in many interesting SM processes. Furthermore, their clean experimental signature makes them very good candidates for triggering purposes. In this document, we discuss how CMS triggers on muons at L1T, highlighting the contribution of the RPC system.

\section{Muon system and legacy Level-1 Muon Trigger}

The CMS muon system [2,3] is made of three complementary detector technologies: the Drift Tubes (DTs) in the barrel, the Cathode Strip Chambers (CSCs) in the endcaps and the Resistive Plate Chambers (RPCs) in both the barrel and endcaps (up to $|\eta|<1.9$ ), as shown in figure 1. The barrel and the endcaps are arranged into four stations, each of them being equipped with RPCs. The first two barrel stations (MB1 and MB2) are equipped with two RPC layers while only one is present in all the other stations. 


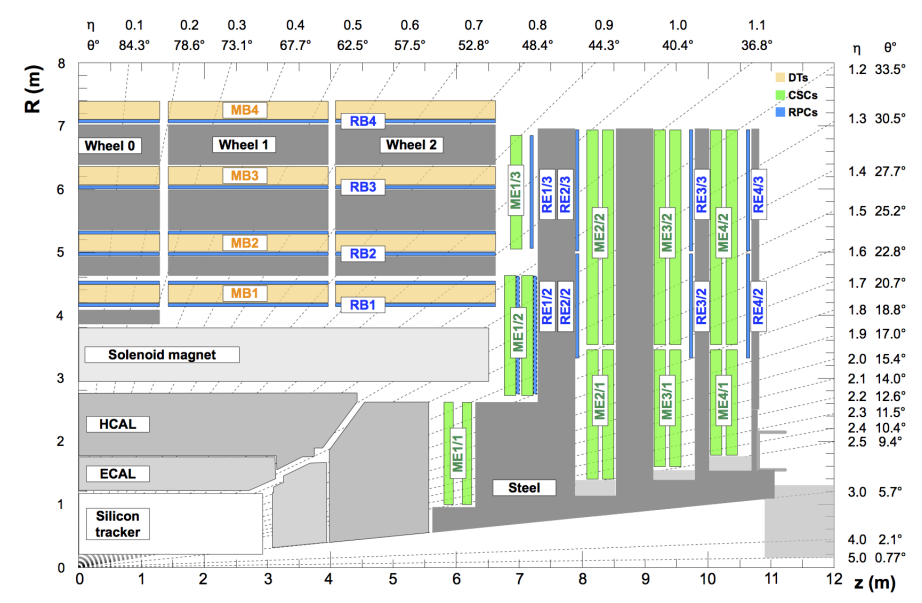

Figure 1. This figure depicts one quadrant of the CMS detector in its Run 2 configuration (from 2015), with the Muon detectors in colour.

CMS already collected proton-proton collision data in two different eras: the so-called Run 1 in 2011 and $2012(\sqrt{s}=7-8 \mathrm{TeV})$ and Run $2(\sqrt{s}=13 \mathrm{TeV})$ between 2015 and 2018. We are now about to start Run 3 data taking which will be followed by the so-called Phase 2 where the instantaneous luminosity will be increased. During Run 1, the CMS Level-1 Muon Trigger was organized based on the three detector technologies in a factorized way [4]. As described in figure 2, three muon track-finders (MTF) were building muon tracks and evaluating muon properties through a single detector technology. The information from different detector was merged after muon candidate reconstruction and duplicates were removed. During the so-called Phase 1 Upgrade (between Run 1 and Run 2), a new way of triggering on muons was deployed to profit from the detector synergies at earlier stages in the reconstruction chain, as described in the next section.
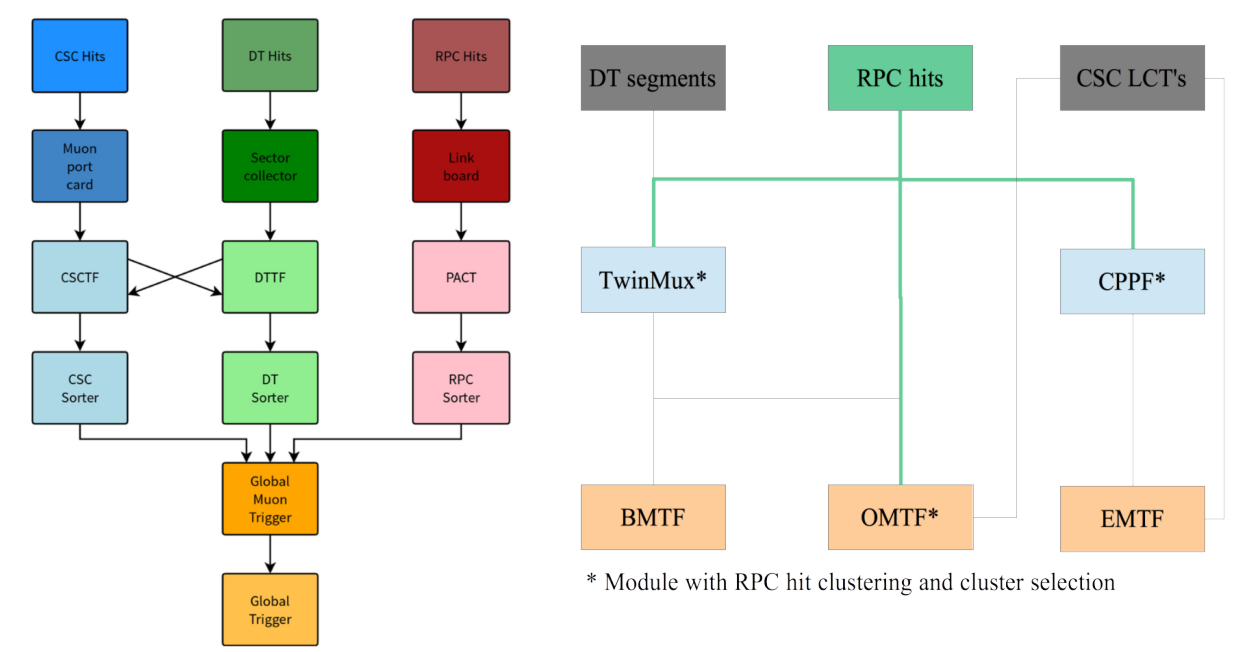

Figure 2. Block diagram of the legacy (left) and Phase 1 (right) CMS Level-1 Muon Trigger architecture. 


\section{RPC in Run 2 Level-1 Muon Trigger}

During Run 2, the track-finder architecture was re-structured from detector-based to region-based as shown in figure 2. We moved from three different track-finders using DT, CSC and RPC hits, respectively, to three track finders reconstructing muon tracks in the barrel $(|\eta|<0.83)$, overlap $(0.83<|\eta|<1.24)$, and endcap $(1.24<|\eta|<2.4)$ region. Each of these track-finders relies on hits from the detectors present in the corresponding region: RPC and DT in the barrel, CSC and RPC in the endcap and the three of them in the overlap region. This allows us to better exploit the detector redundancy and complementarity.

For instance, DT hits are characterized by a better spatial resolution compared to RPC hits while the RPC system shows the highest performance regarding time resolution. In order to exploit this difference, 'super-primitives' are built in the barrel by combining information from both detectors: RPC hits are matched to DT segments and for segments without perfect reconstruction, the bunchcrossing (BX) of the trigger primitive (TP) is assigned from matched RPC information, increasing TP efficiency by $1.4 \%$ as shown in figure 3 (left). In addition to this, RPC only segments are built in case of DT segment absence in the first two stations (MB1 and MB2 are the only stations with two RPC layers allowing us to derive directional information). As shown in the right hand side plot of figure 3, the TP efficiency of MB1 and MB2 is about 4\% higher than in MB3 and MB4 where no RPC only segment can be built.
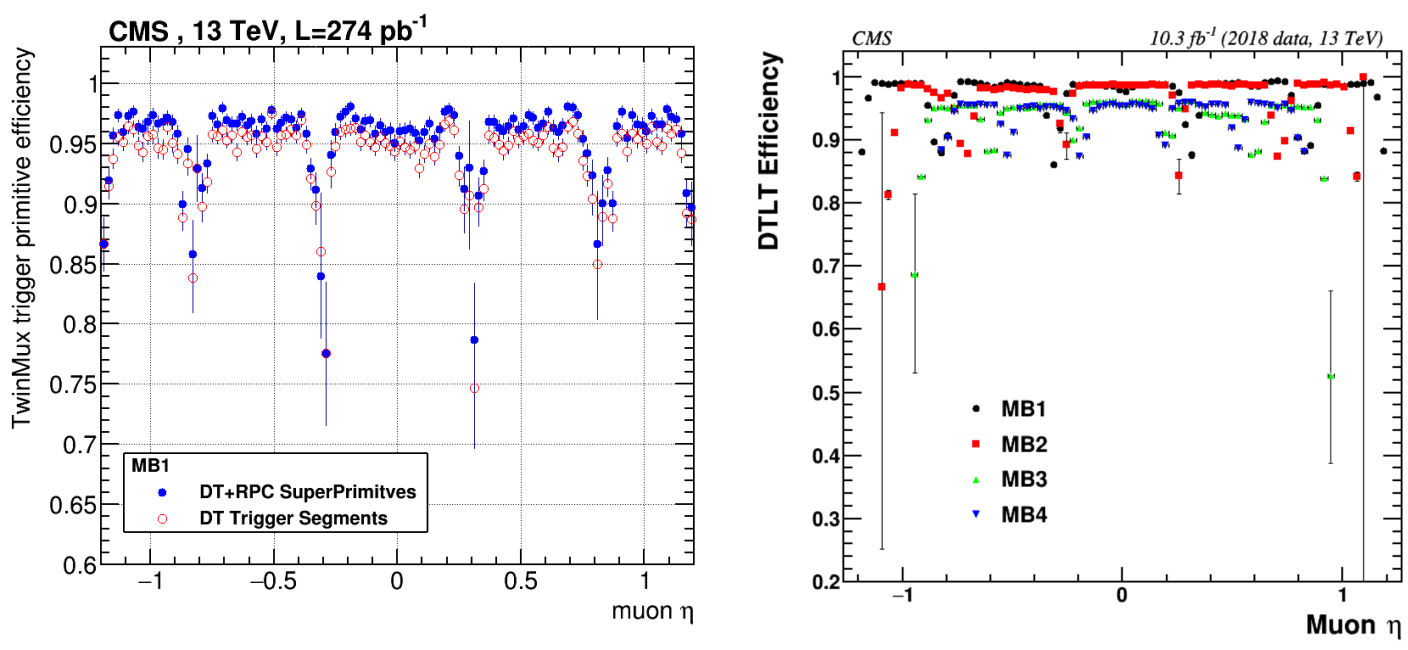

Figure 3. Left: trigger primitive efficiency, for the MB1 station, versus the muon pseudo-rapidity. In red, open circles, the efficiency of DT-only Trigger Primitives. In blue, solid circles, the efficiency of DT+RPC super-primitives. Right: trigger primitive efficiency versus the muon pseudo-rapidity shown for the four stations separately. The RPC only segments are built only in MB1 and MB2 (it requires two RPC layers) which results in an increased efficiency.

The track finder in the overlap region is characterized by a complicated detector geometry and uses the three muon systems to identify muon candidates. The RPC system plays a key role there as it provides 8 measurements for muon trajectory reconstruction (see figure 1). As shown on figure 4, the presence of the RPC system increases the Muon Trigger efficiency by about $15 \%$. 


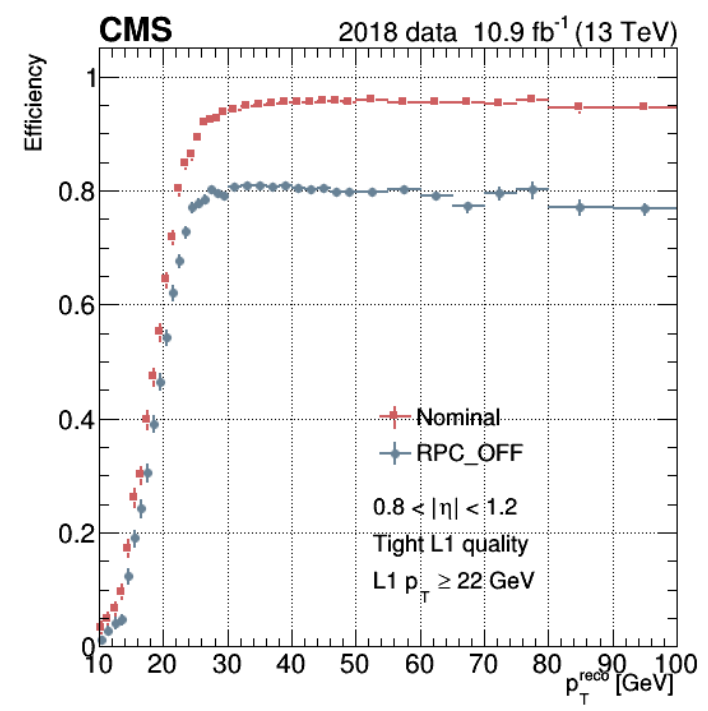

Figure 4. Muon Trigger efficiency vs $\mathrm{p}_{\mathrm{T}}$ for the CMS Level-1 Overlap Muon Track Finder derived from algorithm emulation applied on real data, with and without using the RPC system.

Finally, the endcap muon track finder identifies muon track patterns based on CSC TPs and assigns its kinematic quantities based on a Boosted Decision Tree receiving CSC and RPC TPs information as input.

\section{RPC in Phase 2 Level-1 Muon Trigger}

In view of the High Luminosity LHC (HL-LHC) operations, CMS is preparing a vast upgrade program [5]. Regarding the RPC system, the most relevant features of this upgrade are the increase of the readout frequency from $40 \mathrm{MHz}$ to $640 \mathrm{MHz}$ and the extension of the pseudo-rapidity coverage up to $|\eta|<2.4$ by the introduction of improved RPC (iRPC) chambers in the RE3/1 and RE4/1 regions. These chambers will be equipped with a readout receiving signals from both ends of the strips which will provide the hit position in the radial direction, not accessible in the present system. These chambers will also have thinner gaps ( $1.4 \mathrm{~mm}$ compared to $2 \mathrm{~mm}$ ) with a lower resistivity range (1-3 $\times 10^{10} \Omega \mathrm{cm}$ compared to $1-6 \times 10^{10} \Omega \mathrm{cm}$ ), which will improve the rate capability by about a factor two. Regarding L1T, the key points of the upgrade are the following: the event rate that L1T sends to the HLT will be raised from $100 \mathrm{kHz}$ to $750 \mathrm{kHz}$, the time allowed to take the decision will increase from $3.6 \mu$ s to $12.5 \mu$ s and the whole architecture will be restructured to enable the merging of detector information earlier in the L1T reconstruction sequence.

As during Run 2, RPC and DT information will be merged together to build super-primitives. This will be even more beneficial since the offered redundancy can mitigate to a large extent the expected DT ageing (efficiency loss due to integrated dose) and RPC failures (chamber switched off due to malfunctions). As shown in figure 5, the barrel TP efficiency will remain within 92-99\% if information from both detectors is combined. This situation could further be improved thanks to the new hardware chain allowing us to perform the DT segment building already using RPC information instead of acting on already built DT segments. 


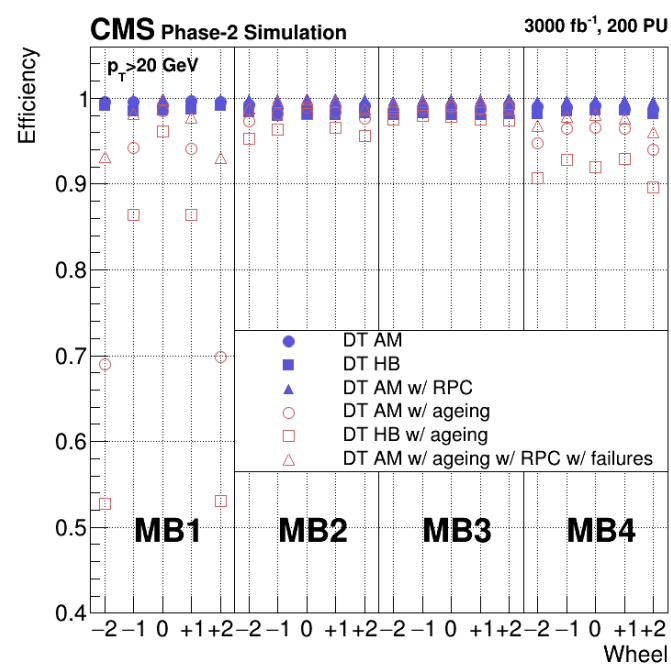

Figure 5. Muon barrel trigger primitive efficiency per station and wheel measured for different reconstruction algorithms: Analytical Method (AM) - with and without enabling the combination of DT+RPC - and Histogram Based Method (HB). The efficiency is computed with respect to offline reconstructed segments matched geometrically with generator level muons with $\mathrm{p}_{\mathrm{T}}>20 \mathrm{GeV}$. Results are computed with and without including detector ageing and failures.

The other key feature of the detector upgrade program is to provide hit timing with more granularity than a BX (25 ns) at L1T. In the barrel, both the DT and RPC systems will be providing sub-BX timing information. As shown in figure 6 (left), a gain of 15(30)\% in time resolution is obtained when the time measurement is coming from the RPC detector without(with) DT ageing considered. In the endcap, the RPCs and iRPCs will be the only systems providing sub-BX timing information in the L1T. The expected time resolution from endcap muon TPs is shown in figure 6 (right). It is slightly better than the barrel one due to the fact that strips are shorter in the RPC endcap, lowering the smearing from unknown signal propagation along the strip. This sub-BX timing will be used to introduce a new capability to trigger based on the time-of-flight opening thus sensitivity to e.g. Heavy Stable Charged Particles which behave as muons moving at velocities significantly lower than the speed of light. Figure 7 shows how such a dedicated trigger using time-of-flight can bring efficiency back to $\sim 90 \%$ for velocities down to $\beta=0.3$ while prompt Muon Triggers start being inefficient at $\beta=0.7$. This sub-BX timing will also be useful to prevent us from building muon tracks with hits that are not compatible with each other regarding their timing, improving thus the $\mathrm{p}_{\mathrm{T}}$ resolution and lowering the fake rates.

\section{Conclusion}

The Level-1 Muon Trigger is crucial for the CMS physics program and significantly relies on the RPC system. It has been re-designed to best exploit the redundancy and complementarity of all the different detector technologies making it a very high performance and robust system. The Phase 2 upgrade program ensures that we will keep very good performances up to the end of the HL-LHC 

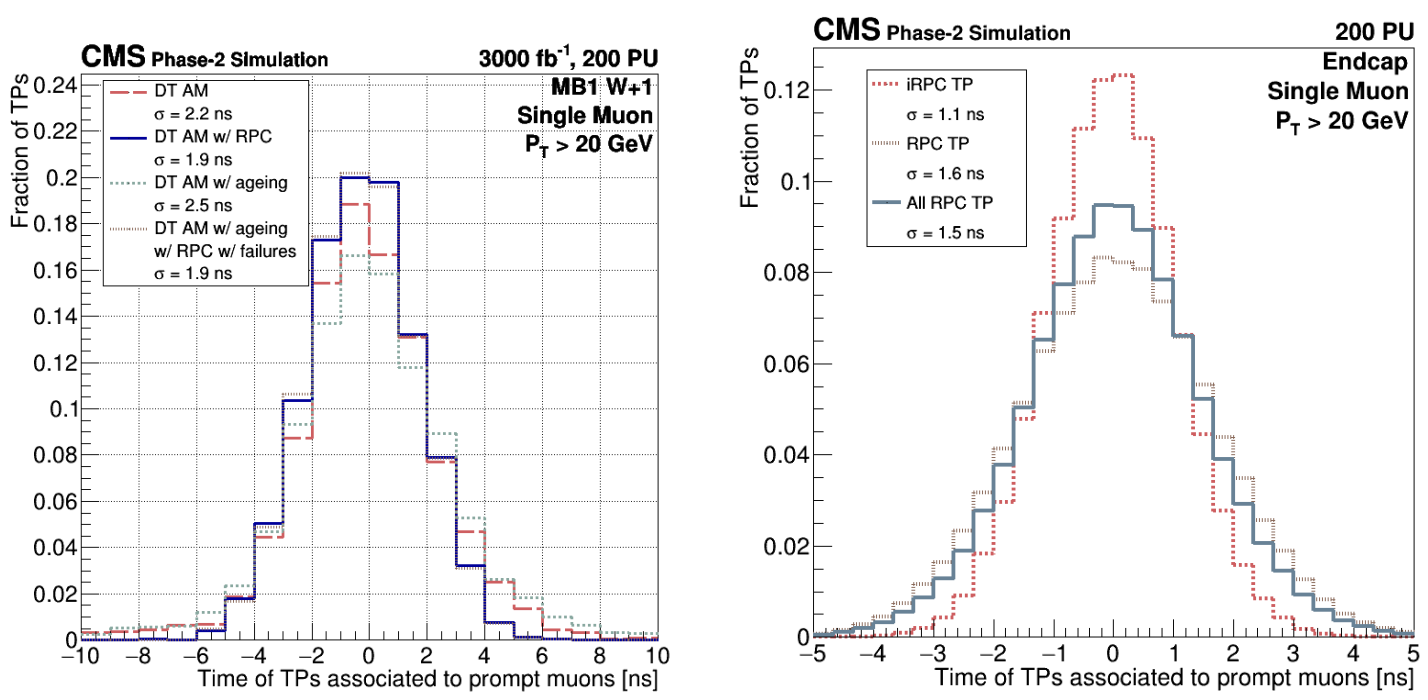

Figure 6. Left: timing distribution of muon TPs generated with and without enabling the combination into DT+RPC SPs. Results are computed for muons with $\mathrm{p}_{\mathrm{T}}>20 \mathrm{GeV}$ with and without including detector ageing and failures. Right: time distribution of the endcap (i)RPC TPs associated to a generated muon track with $\mathrm{p}_{\mathrm{T}}>20 \mathrm{GeV}$. The solid blue line, labelled as 'All RPC TP', represents the distribution obtained without separating RPC and iRPC TPs.

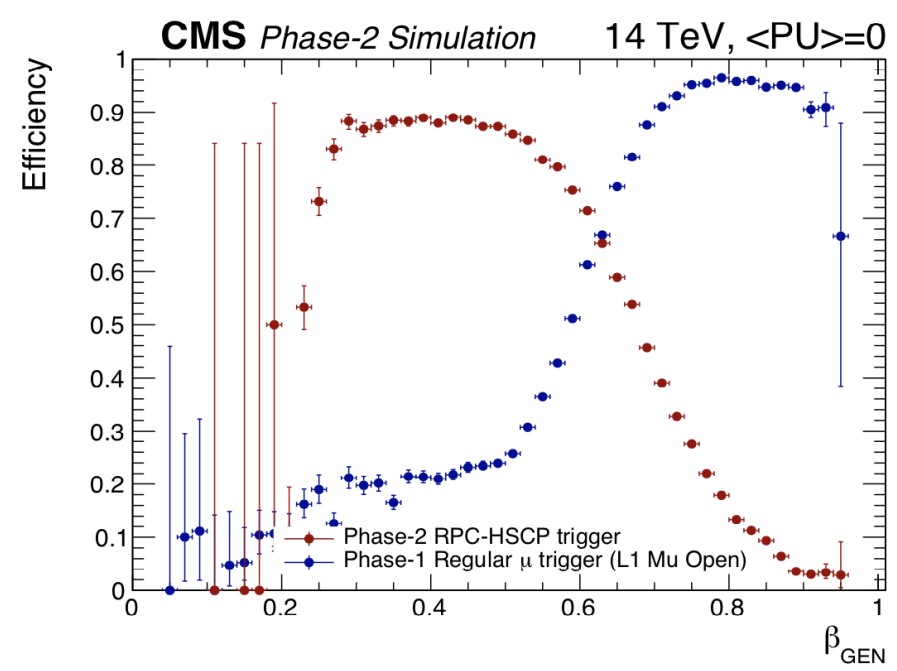

Figure 7. Left: Muon barrel trigger primitive efficiency measured for two different DT reconstruction algorithm: Analytical Method (AM) - with and without enabling the combination of DT+RPC - and Histogram Based Method (HB). The efficiency is computed with respect to offline reconstructed segments matched geometrically with generator level muons with $\mathrm{p}_{\mathrm{T}}>20 \mathrm{GeV}$. Results are computed with and without including detector ageing and failures.

operations and brings new possibilities to further increase synergies among detectors and extend the physics program thanks to new exotic trigger paths. 


\section{References}

[1] CMS collaboration, The CMS Experiment at the CERN LHC, 2008 JINST 3 S08004.

[2] CMS collaboration, The CMS muon project: Technical Design Report, Technical Design Report CMS, CERN-LHCC-97-032, CERN, Geneva (1997).

[3] CMS collaboration, Performance of the CMS muon detector and muon reconstruction with proton-proton collisions at $\sqrt{s}=13 \mathrm{TeV}, 2018$ JINST 13 P06015 [arXiv : 1804. 04528].

[4] D. Rabady, J. Ero, G. Flouris, J. Fulcher, N. Loukas, E. Paradas et al., Upgrade of the CMS Muon Trigger system in the barrel region, Nucl. Instrum. Meth. A 845 (2017) 616.

[5] CMS collaboration, The Phase-2 Upgrade of the CMS Muon Detectors, Tech. Rep. CERN-LHCC-2017-012, CMS-TDR-016, CERN, Geneva (2017). 